\title{
Search for squarks and gluinos in final state with jets and missing transverse momentum with the ATLAS detector at the LHC
}

\author{
Manfredi Ronzani* On behalf of the ATLAS Collaboration \\ Albert-Ludwigs-Universitaet Freiburg (DE) \\ E-mail: manfredi.ronzaniecern.ch
}

\begin{abstract}
This proceeding summarises results on inclusive searches for squarks and gluinos in final state containing jets, missing transverse momentum and no leptons ( $0 \mathrm{~L})$. The first search presented uses the full dataset recorded in 2012 by the ATLAS experiment in $\sqrt{s}=8 \mathrm{TeV}$ centre-of-mass energy pp collisions at the LHC, corresponding to a total luminosity of $20.3 \mathrm{fb}^{-1}$. No significant excess above the Standard Model expectations is observed and several interpretations in the context of simplified phenomenological supersymmetric models are shown. Moreover, sensitivity studies for gluino pair production with a full simulation of the ATLAS detector at a centre-of-mass energy of $13 \mathrm{TeV}$ are presented. In the end, the first Run2 data distributions in regions dominated by the $\mathrm{W}+$ jets, $\mathrm{Z}+\mathrm{jets}$ and combined top events in the same final state are shown. Data are collected by the ATLAS experiment at $\sqrt{s}=13 \mathrm{TeV}$ in 2015, corresponding to an integrated luminosity of 78 $\mathrm{pb}^{-1}$.
\end{abstract}

The European Physical Society Conference on High Energy Physics

22-29 July 2015

Vienna, Austria

* Speaker. 


\section{Introduction and Motivation}

Supersymmetry (SUSY) is a theoretically favoured candidate for physics beyond the Standard Model (SM). Many Supersymmetric models predict squarks $(\tilde{q})$ and gluinos ( $\tilde{g})$ that could be accessible at the LHC, due to the large cross sections. In SUSY theories where R-parity conservation is assumed, squarks and gluinos decay in cascades producing SM particles and lighter SUSY particles. The Lightest Supersymmetric Particle (LSP) is eventually stable and weakly interacting and it is supposed to be undetected resulting in a signature of missing transverse momentum $\left(\mathrm{E}_{\mathrm{T}}^{\mathrm{miss}}\right)$. This proceeding summarizes the most recent results in the search for squarks and gluinos in final state with jets and $\mathrm{E}_{\mathrm{T}}^{\mathrm{miss}}$ with a veto on leptons applied (0L analysis), in order to be orthogonal to other ATLAS[1] searches.

\section{Run1 Analysis and Interpretations}

\subsection{Event Selection and Backgroud Determination}

The analysis on Run1 data [2] uses a combined jet $+\mathrm{E}_{\mathrm{T}}^{\mathrm{miss}}$ trigger which is fully efficent in events with at least one jet with transverse momentum $p_{\mathrm{T}}$ above $130 \mathrm{GeV}$ and $\mathrm{E}_{\mathrm{T}}^{\mathrm{miss}}$ above 160 $\mathrm{GeV}$. Events with jet multiplicity from 2 to 6 are selected and the other jets must have $p_{\mathrm{T}}$ above 60 $\mathrm{GeV}$. The main variable used to discriminate the signal from the background is the effective mass $\mathrm{m}_{\text {eff }}$, defined as the scalar sum of the $p_{\mathrm{T}}$ of the leading $\mathrm{n}$-jets in the $\mathrm{n}$-jets channel and the $\mathrm{E}_{\mathrm{T}}^{\mathrm{miss}}$. In order to suppress multijet events where the $\mathrm{E}_{\mathrm{T}}^{\mathrm{miss}}$ comes from a mis-measurement of the jet energy, a cut on the angular separation between jets and missing transverse momentum is applied.

In this analysis 15 inclusive signal regions (SRs) are defined depending on the minimal number of jets present in the final state and on the final selection consisting of two cuts on $m_{\text {eff }}$ and on the ratio $\mathrm{E}_{\mathrm{T}}^{\text {miss }} / \mathrm{m}_{\mathrm{eff}}\left(\mathrm{N}_{\mathrm{j}}\right)$ or $\mathrm{E}_{\mathrm{T}}^{\text {miss }} /{\sqrt{\mathrm{H}_{\mathrm{T}}}}^{1}$. The lower jet multiplicity SRs are optimized on models with squark pair production with short decay chain while the SRs requiring higher jet multiplicity focus on the gluino pair production.
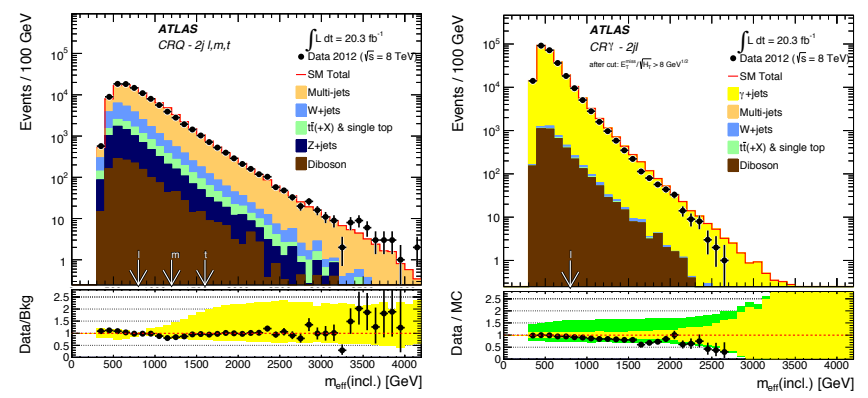

Figure 1: Observed $m_{\text {eff }}$ distributions for the 2-jets control regions CRQ (left) and CRY (right) [2].

The main Standard Model (SM) background processes contributing to the final event yield are: $\mathrm{Z}+$ jets, where $\mathrm{Z}$ decays into neutrinos, $\mathrm{W}+\mathrm{jets}$ where the lepton produced by the $\mathrm{W}$ decay is faking a jet, the Top pairs and Single Top processes, multijet and diboson production. In order to estimate

\footnotetext{
${ }^{1} \mathrm{H}_{\mathrm{T}}$ is defined as the scalar sum of the transverse momenta of all $p_{\mathrm{T}}>40 \mathrm{GeV}$ jets
} 
Table 1: Control regions used in the analysis. Also listed are the main targeted backgrounds in the SR in each case, the process used to model the background, and the main CR requirement(s) used to select this process.

\begin{tabular}{lccc}
\hline CR & SR background & CR process & CR selection \\
\hline CR $\gamma$ & $Z(\rightarrow v v)+$ jets & $\gamma+$ jets & Isolated photon \\
CRQ & Multi-jets & Multi-jets & SR with reversed requirements on (i) $\Delta \phi\left(j e t, E_{\mathrm{T}}^{\text {miss }}\right)$ \\
& & & and (ii) $E_{\mathrm{T}}^{\text {miss }} / m_{e f f}\left(N_{\mathrm{j}}\right)$ or $E_{\mathrm{T}}^{\text {miss }} / \sqrt{H_{\mathrm{T}}}$ \\
CRW & $W(\rightarrow \ell v)+$ jets & $W(\rightarrow \ell v)+$ jets & $30 \mathrm{GeV}<m_{\mathrm{T}}\left(\ell, E_{\mathrm{T}}^{\text {miss }}\right)<100 \mathrm{GeV}, b$-veto \\
CRT & $t \bar{t}$ and single- $t$ & $t \bar{t} \rightarrow b \bar{b} q q^{\prime} \ell v$ & $30 \mathrm{GeV}<m_{\mathrm{T}}\left(\ell, E_{\mathrm{T}}^{\text {miss }}\right)<100 \mathrm{GeV}, b$-tag \\
\hline
\end{tabular}

the first four sources, four control regions (CRs) are defined for each SR. Each control region is enriched in one of the background sources and they are optimised to have enough statistics and negligible contribution for the SUSY signal contamination (see Figure 1). They are designed to be orthogonal to SRs and their definitions are given in Table 1. The SM background estimates for the SRs are obtained using the observed numbers of events in CRs via a likelihood fit which takes in account the ratios of expected event counts from each background process between the SR and each CR, and between CRs. The ratios are called "Transfer Factors". The minor backgrounds such as diboson events are estimated directly from the MonteCarlo (MC) simulation.

\subsection{Results and Interpretations}

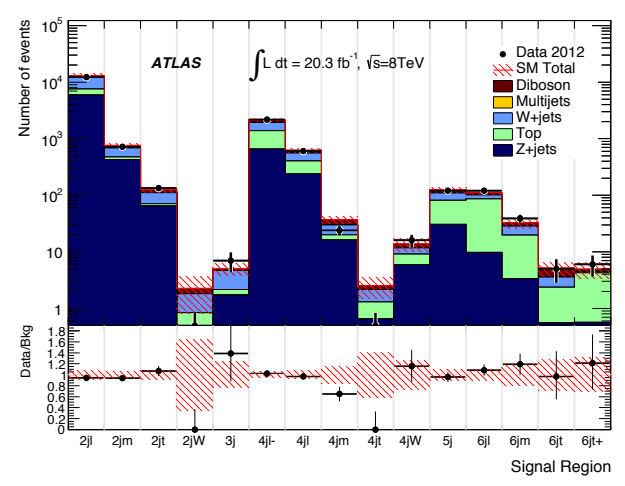

Figure 2: Comparison of the observed and expected event yields as a function of signal region [2].

For each signal region the number of SM events is estimated using a likelihood fit. No significant excess is observed above the SM expectations. The results are then interpreted in terms of limits on the parameter space of different SUSY models. For each model point, the best performing SR in terms of expected sensitivity is used. The $95 \% \mathrm{CL}$ exclusion regions are derived using a log-likelihood ratio test in combination with the CLs prescription. Limits for simplified models in the context of the phenomenological MSSM (Minimal Superymmetric extension of the SM), in which only production of gluino or first- and second- generation of squarks with direct decay to lightest $\tilde{\chi}_{1}^{0}$ are considered, are shown in Figure 3 (left). The results are also interpreted for two sets 
of simplified models involving the pair production of gluinos decaying directly into two quarks and lighest $\tilde{\chi}_{1}^{0}$ and shown in Figure 3 (right).
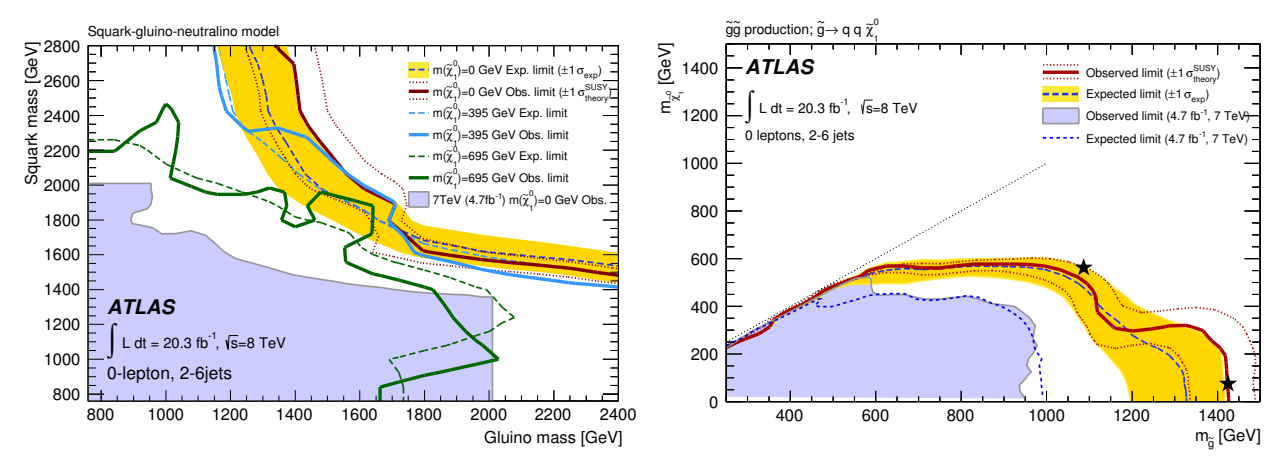

Figure 3: Left: Exclusion limits for a simplified pMSSM scenario with only strong production of gluinos and first- and second-generation squarks (of common mass), with direct decays to quarks and lightest neutralinos. Right: Three values of the lightest neutralino mass are considered: $m_{\tilde{\chi}_{1}^{0}}=0,395$ and $695 \mathrm{GeV}$. Exclusion limits for direct production of gluino pairs with decoupled squarks. The dashed lines show the expected limits at $95 \% \mathrm{CL}$, with the light (yellow) band indicating the $1 \sigma$ experimental and background-only theory uncertainties. [2].

\subsection{Further Interpretations and Combinations}

The results of the Run1 analysis have been interpreted in terms of several other SUSY models. Some of them present final states which are also accessible by other analyses performed by ATLAS. Those analyses can be statistically combined with the analysis presented above in order to increase the exclusion reach in particular SUSY models. The combination requires that the two analyses are designed to be statistically independent in their SRs and CRs definitions and they have to provide comparable sensitivities on the model. The conditions are satisfied for a combination [3] of the one-lepton (1L) and $0 \mathrm{~L}$ searches, which differ on the base of the request of an isolated lepton in final states $(1 \mathrm{~L})$ or the application of an explicit lepton-veto (0L). The statistical combination is obtained from the individual likelihoods of the analyses involved. The correlated uncertainties in the combination procedure are the luminosity uncertainty, the uncertainty on the SUSY cross-sections, b-tagging uncertainties, and the jet energy scale and resolution and $\mathrm{E}_{\mathrm{T}}^{\mathrm{miss}}$ related uncertainties.

In Figure 4 (left) is shown the result of the combination of the $1 \mathrm{~L}$ and $0 \mathrm{~L}$ analyses for the simplified model with one-step decays of the pair-produced gluinos. The model assumes that these particles decay via the $\tilde{\chi}_{1}^{ \pm}$into a $\mathrm{W}$ boson and the $\tilde{\chi}_{1}^{0}$. The limits obtained improve the results of the separate analyses, reaching higher $\tilde{\chi}_{1}^{0}$ mass and approximately $50 \mathrm{GeV}$ higher gluino mass for massless neutralinos.

In the context of the $0 \mathrm{~L}$ analysis, two new signal regions (respectively named $0 \mathrm{~L} \_4 \mathrm{jt}+$ and 0L_5jt) have been built and optimized [3]. The purpose is to interpret the measurements with a phenomenological MSSM model in which only the left-handed squarks of the first two generations, the two lightest neutralinos $\tilde{\chi}_{2}^{0}$ and $\tilde{\chi}_{1}^{0}$, and the lightest chargino $\tilde{\chi}_{1}^{ \pm}$are assumed to be within kinematic reach. Left-handed squarks can be pair produced only via t-channel gluino exchange and they can undergo a one-step decay: $\tilde{q}_{L} \rightarrow q+\tilde{\chi}_{2}^{0} \rightarrow q+Z / h+\tilde{\chi}_{1}^{0}$ or $\tilde{q}_{L} \rightarrow q+\tilde{\chi}_{1}^{ \pm} \rightarrow q+W^{ \pm}+\tilde{\chi}_{1}^{0}$, 

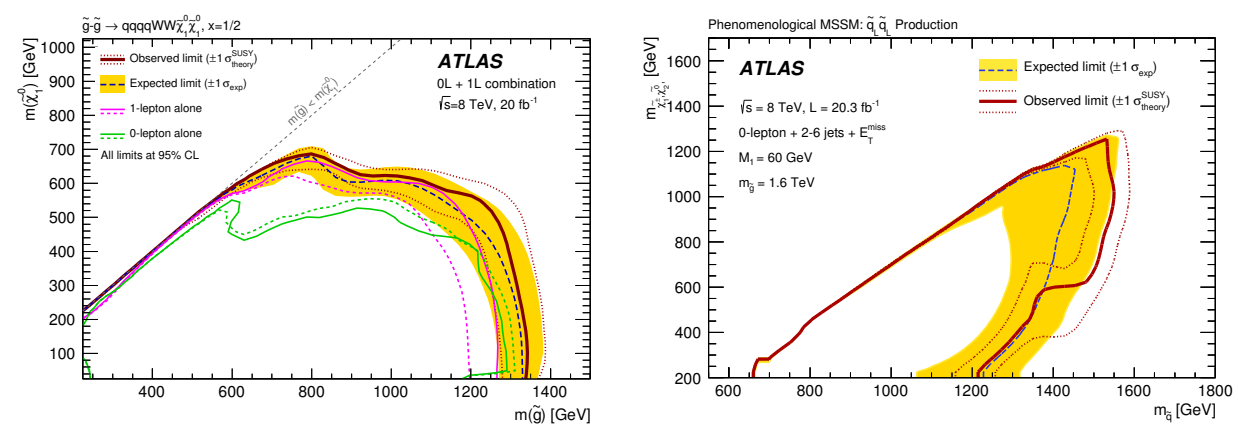

Figure 4: Observed and expected exclusion limits for simplified models of gluino-pair production with one-step decays via the $\tilde{\chi}_{1}^{ \pm}$into a $\mathrm{W}$ boson and the $\tilde{\chi}_{1}^{0}$. The mass of the $\tilde{\chi}_{1}^{ \pm}$is chosen to be between the $m_{\tilde{g}}$ and the $m_{\tilde{\chi}_{1}^{0}}$. The individual limits from the $0 \mathrm{~L}$ and the $1 \mathrm{~L}$ analyses are overlaid in green and magenta, respectively (left). 95\% CL exclusion limits in the pMSSM considered in the search for left-handed squarks, with mass parameters M1 and M2, which are associated with the bino and wino masses, respectively. Here $\mathrm{M} 1$ is set to $60 \mathrm{GeV}$ and M2 is varying (right) [3].

where the lightest Higgs boson $h$ is assumed to have the SM decay branching fractions, and its mass is set to $125 \mathrm{GeV}$. In Figure 4 (right) limits for $m_{\tilde{g}}=1600 \mathrm{GeV}$, as obtained from the $0 \mathrm{~L}$ analysis with the additional SRs are shown. As expected, a large range of squark masses (up to $1500 \mathrm{GeV}$ ) and $\tilde{\chi}_{1}^{ \pm} / \tilde{\chi}_{2}^{0}$ masses (up to $1150 \mathrm{GeV}$ ) can be excluded.

\section{Expected Sensitivity and First Look at the Run2 13 TeV Data}

Before the start of LHC Run2, the expected sensitivities for the $0 \mathrm{~L}$ analysis with the early Run2 dataset based on fully simulated samples have been provided [4]. The results rely heavily on the approaches described in Section 2. The SRs are reoptimised for the $13 \mathrm{TeV}$ centre-of-mass energy data and rely on MC predictions for the signal and SM backgrounds. As the sensitivity measure, signal significance is calculated on the discovery p0 values from the background-only hypothesis test for a counting experiment. Various assumptions on the integrated luminosity (1, 2,5 , or $10 \mathrm{fb}^{-1}$ ) and on the uncertainty for the total background prediction (the same level as the total background uncertainty observed in Run-1, or a more conservative hypothesis for early data taking) are evaluated. For the gluino-pair production incorporating only a gluino and the lightest neutralino, a gluino with a mass of $1.5 \mathrm{TeV}$ could be seen with a significance of $3 \sigma$ with an integrated luminosity of $5 \mathrm{fb}^{-1}$, if the background can be understood within an uncertainty of less than $20 \%$.

After the start of the LHC Run2, a first look at the regions dominated by the main SM background processes in the 0L analysis has been provided for the EPS 2015 conference [5]. The CRs select events with at least one high-purity isolated lepton and are designed to normalise Monte Carlo predictions to data. This study relies heavily on the search designed for the analysis of the 8 $\mathrm{TeV}$ data and described in Section 2. The dataset used in this analysis was collected in 2015 with the LHC colliding $6.5 \mathrm{TeV}$ proton beams with $50 \mathrm{~ns}$ bunch spacing. The total integrated luminosity after the data-quality requirements is of $78 \mathrm{pb}^{-1}$. The details on the event selection can be found in 


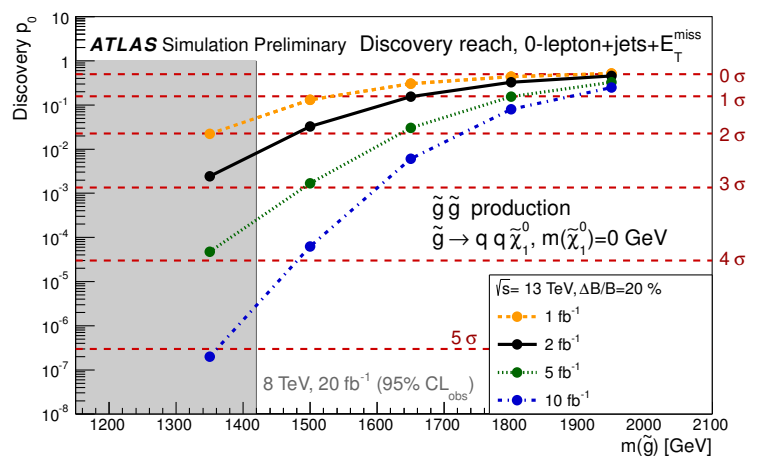

Figure 5: Discovery p0 values as a function of the gluino mass in the gluino-pair production models with $m_{\tilde{\chi}_{1}^{0}}=0$, at a given integrated luminosity of $1,2,5$, or $10 \mathrm{fb}^{-1}$ (from inner to outer lines) with a hypothesis of $20 \%$ uncertainties on the total background prediction [4].

[5]. The MC simulation is found to well describe the data demonstrating the good level of control over these backgrounds to searches for Supersymmetry, as shown in Figure 6.
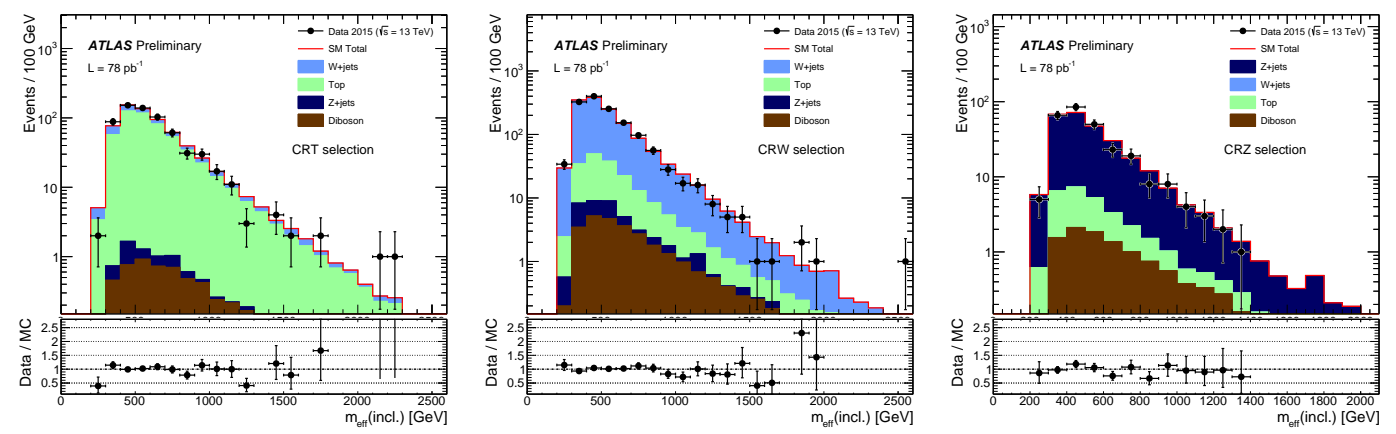

Figure 6: Distribution of the observed effective mass, $m_{\mathrm{eff}}$ (inlc.), calculated as the scalar sum of the transverse momenta of jets with $p_{\mathrm{T}}>40 \mathrm{GeV}$ and $\mathrm{E}_{\mathrm{T}}^{\mathrm{miss}}$ in control regions CRT (left), CRW (centre) and CRZ (right)[5].

\section{References}

[1] ATLAS Collaboration, JINST 3 S08003 (2008).

[2] ATLAS Collaboration, Search for squarks and gluinos with the ATLAS detector in final states with jets and missing transverse momentum using $\sqrt{s}=8 \mathrm{TeV}$ pp collision data, JHEP 09 (2014) 176, doi: 10.1103/PhysRevD.90.052008, arXiv:1407.0608[hep-ex].

[3] ATLAS Collaboration, Summary of the searches for squarks and gluinos using $\sqrt{s}=8 \mathrm{TeV} \mathrm{pp}$ collisions with the ATLAS experiment at the LHC, arXiv:1507.05525 [hep-ex].

[4] ATLAS Collaboration, Expected sensitivity studies for gluino and squark searches using the early LHC 13 TeV Run-2 dataset with the ATLAS experiment, ATL-PHYS-PUB-2015-005 (2015).

[5] ATLAS Collaboration, First look at pp collisions data at $\sqrt{s}=13 \mathrm{TeV}$ in preparation for a search for squarks and gluinos in final states with jets and missing transverse momentum with the ATLAS detector, ATL-PHYS-PUB-2015-028 (2015). 